\title{
Successful treatment of narcolepsy and cataplexy: A review
}

\author{
B Lockrane MBChB, P Bhatia MBBS MRCP, Robin Gore MBChB MD MRCP
}

B Lockrane, P Bhatia, R Gore. Successful treatment of narcolepsy and cataplexy: A review. Can Respir J 2005;12(4):225-227.

A 25-year-old woman with narcolepsy and cataplexy was treated with well-recognized drugs for these conditions. Because she did not respond, a decision was made to treat her with sodium oxybate.

Key Words: Cataplexy; Narcolepsy; Sodium oxybate

\section{Réussite du traitement de la narcolepsie et de la cataplexie : Survol}

Une femme de 25 ans atteinte de narcolepsie et de cataplexie a été soignée au moyen de médicaments bien établis pour le traitement de ces maladies. Compte tenu de l'absence de réponse, il a été décidé de la traiter au moyen d'oxybate de sodium.

\begin{abstract}
A 25-year-old woman with no significant past medical history was referred by her general practitioner to the sleep clinic in North Manchester General Hospital (United Kingdom) with an 18-month history of terrifying, vivid nightmares on most nights. She would sometimes wake from these nightmares to find herself paralyzed and trying to shout out but making no sound. She became frightened of going to sleep at night, found no relief with sleeping tablets, and fell asleep easily during the day because she was constantly tired. She was in a stable relationship with two children, had no history of anxiety or depression, smoked 15 cigarettes per day, drank alcohol occasionally, and didn't abuse any drugs.
\end{abstract}

Her symptoms were classical of narcolepsy. A multiple sleep latency test (MSLT) measurement was consistent with the diagnosis; latencies to rapid eye movement (REM) sleep were recorded at $8 \mathrm{~min}$ and $10 \mathrm{~min}$ in two of four recorded naps. Genetic tests were positive for HLA-DRB1*15 and HLA-DQB1*0602.

Over the course of the next six years, the patient went through all of the standard therapies for narcolepsy, demonstrating an unusual overall resistance to treatment. Her progress was monitored using the Epworth Sleepiness Score (ESS), which remained consistently between 21 and 24. Her initial treatment options were limited because she was pregnant at the time, but she was started on amitriptyline at a dose of $50 \mathrm{mg}$ at night in an attempt to suppress the REM sleeprelated phenomena. She experienced a significant initial improvement in her symptoms, particularly with nightmares. Symptoms of excessive daytime sleepiness continued to present a very significant problem, particularly with the birth of her new son. When she was caring for her two other young children, she had instances of falling asleep uncontrollably when she was responsible for their safety.

Modafinil, a nonamphetamine stimulant, was started initially at $100 \mathrm{mg}$ in the morning and at midday; this was later increased to a maximum dose of $300 \mathrm{mg}$ twice daily with no effect. She was later commenced on dexamphetamine at $10 \mathrm{mg}$ daily in a split dose. She found this to be quite effective, and her ESS was recorded at 18/24. As time went on, the patient became tolerant to its effects, and the dose had to be increased until she was taking $50 \mathrm{mg}$ daily. Although she was able to maintain wakefulness for the course of the day, she would succumb to sleep in the early evening, from which she would feel so refreshed that, in combination with the dexamphetamines, she would then be unable to sleep for most of the night. She later found that she was falling asleep during the day in spite of the dexamphetamines, and her ESS slipped back to between 21 and 24

The ensuing years were a roller coaster of attempts to wean her off the dexamphetamines, accompanied by a deterioration in waking and sleeping patterns, with fluctuations in other sleep-related phenomena. Two years after the initial diagnosis, she started to experience cataplectic attacks, or 'sleep attacks'. These attacks were usually triggered by a strong emotion such as laughter, and as a result, she would suddenly become paralyzed and collapse. Although she appeared to be unconscious, she would actually be audibly aware of her surroundings, but would be unable to express any movement or speak. She reported multiple attacks in quick succession, in which the same emotional trigger would paralyze her each time. They could last up to $2 \mathrm{~h}$ on some occasions and, at their peak, she was experiencing up to 10 per day, with significant repercussions to her life. She was now receiving a disability living allowance, and her driving licence had been revoked. Paroxetine $20 \mathrm{mg}$ and clomipramine up to a dose of $60 \mathrm{mg}$ were both tried in an attempt to control the cataplectic attacks, with no benefit.

In December 2003, a named person licence was obtained for the patient to have sodium oxybate. She initially started on a low dose of $2.25 \mathrm{~g}$ at bedtime followed by another $2.5 \mathrm{~g} 4 \mathrm{~h}$ later. Although there was no improvement in the cataplectic attacks, she experienced less drowsiness during the day, and her ESS was recorded at $17 / 24$. An increase of the dose to $7.5 \mathrm{~g}$ 
resulted in a significant improvement in sleepiness (ESS 8/15) and the cessation of cataplectic attacks. She reported occasional urinary incontinence as the only side effect of the treatment, with no nausea, dizziness or sleepwalking. She is under regular follow-up and remains well-controlled.

\section{NARCOLEPSY: A REVIEW}

It was Gélineau who first used the word 'narcolepsy' in 1880 to describe a pathological condition characterized by irresistible episodes of sleep of short duration recurring at close intervals. He also described the association of 'astasias' (falls) with narcolepsy, which was later referred to as cataplexy (1). In the 1930s, Daniels (2) emphasized the association among daytime sleepiness, cataplexy, sleep paralysis and hypnagogic hallucinations.

The prevalence of narcolepsy varies among countries due to genetic factors. Men are affected more often than women, and age at onset varies from childhood to the fifth decade, with a peak in the second decade. Up to $50 \%$ of the cases are preceded by an abrupt change in sleep-wake schedule or severe psychological stress (death of a relative or divorce). The majority of patients with definite cataplexy share a specific HLA allele on chromosome six (DQB1*0602); however, this may be present in up to $38 \%$ of the general population (3). A second gene involved in narcolepsy has been identified. The hypocretin (orexin) gene is involved in the control of the hypocretin receptor. Narcoleptic patients lack hypocretin in the cerebrospinal fluid. Normally, two hypothalamic neural pathways mediate the regulation of sleep versus wake. A gammaaminobutyric acid system arising in the ventrolateral preoptic nucleus promotes sleep by inhibiting the ascending arousal system. The hypocretin pathway promotes waking by activating the ascending arousal system.

Diagnostic criteria within The International Classification of Sleep Disorders: Diagnostic and Coding Manual (4) includes the classic tetrad for narcolepsy: excessive daytime sleepiness, cataplexy, sleep paralysis and hypnagogic hallucinations. Automatic behaviours and disrupted nighttime sleep are also commonly seen. It must be remembered that not all of these symptoms are present in all patients.

Unwanted episodes of sleepiness recur several times per day; they may occur while the subject is fully involved in a task such as eating a meal or speaking on the telephone. Each episode may last from a few minutes (if the subject is in an uncomfortable position) to up to $1 \mathrm{~h}$, with rare instances lasting over $1 \mathrm{~h}$. The subject wakes up refreshed from these naps. These episodes can result in poor performance at work.

Cataplexy is an abrupt and reversible loss of muscle tone most frequently elicited by emotions such as laughter, anger, fear or surprise. It may involve certain muscles or the entire voluntary musculature. The severity and extent of cataplectic attacks can vary from complete powerlessness in the entire voluntary musculature to transient weakness in a localized group of muscles. During an attack, speech may be impaired and respiration may become irregular. Patients may sustain serious injuries during an attack, and patients may describe these attacks as clumsiness if it involves the upper limbs and results in the dropping of cups, plates, etc. Short cataplectic attacks are most commonly seen and easily missed by even skilled physicians. Cataplexy is associated with an inhibition of the monosynaptic $\mathrm{H}$ reflex and multisynaptic tendon reflexes.
Sleep paralysis is a terrifying experience that occurs in narcoleptics when falling asleep or awakening. During an episode of sleep paralysis, patients are unable to move their limbs, speak or even breathe deeply, although he or she may be fully aware of the condition. Terrifying hallucinations may accompany an episode of sleep paralysis.

Hypnagogic hallucinations are unpleasant auditory or visual hallucinations that accompany sleep paralysis. The visual hallucinations consist of coloured circles, parts of objects, or images of animals or people. Auditory hallucinations may range from sounds to an elaborate melody. Other interesting hallucinations include cenesthopathic feelings (feeling of rubbing, light touch) and feelings of levitation or extracorporeal experiences, where patients report that they are above the bed and can see their body below.

Nighttime sleep is often interrupted by repeated awakenings accompanied by terrifying dreams. Patients may complain of trouble falling asleep at night, although they may fall asleep repeatedly during the daytime.

The diagnosis of narcolepsy is a two-stage procedure. First, it is essential to confirm excessive daytime sleepiness (EDS) and then, second, confirm that it is due to narcolepsy. EDS can be assessed by questionnaires such as the Stanford Sleepiness Scale or the ESS. These, however, are subjective tools and should be followed by objective evidence of EDS. At the time of the sleep diagnostic studies, the patient should follow a normal daily routine with at least $8 \mathrm{~h}$ nocturnal sleep and should not be on any sedating drugs. A polysomnogram should be performed at night, and other causes of EDS, such as obstructive sleep apnea, should be excluded or treated before performing the MSLT. The MSLT is a series of nap opportunities (four to six) presented at $2 \mathrm{~h}$ intervals. The electrophysiological parameters needed to detect sleep onset and score sleep stages are recorded. Sleep latency, defined as the time elapsed from the start of the test to sleep onset in normal controls, ranges from $10 \mathrm{~min}$ to $20 \mathrm{~min}$. A sleep latency of less than $5 \mathrm{~min}$ confirms pathological sleepiness. In addition to short sleep latency, REM sleep onset on three of the naps confirms narcolepsy.

\section{TREATMENT}

The aim of treatment is to control the symptoms and allow the patient to have a full family and professional life. Drug treatment may be lifelong; therefore, it must balance disease control and side effects.

\section{Nonpharmacological measures}

Nonpharmacological measures include regular timing of nocturnal sleep, avoidance of shift work and alcohol, and regular, strategically timed naps. In addition, career counselling is important because both patients and their employers should be aware of the risks of performing certain jobs; if employers recognize the importance of short 15 min to 20 min naps during the daytime, then the patient can be a valuable employee.

\section{Drug treatment for EDS}

Dexamphetamine: Dexamphetamine, a central nervous system stimulant, has been used in patients with narcolepsy to treat EDS since 1935 (5). It can be combined with short naps to reduce EDS. It is metabolized in the liver, and any hepatic dysfunction will affect its metabolism. Besides dependence and psychotic states, it is associated with irritability, nervousness, headache, palpitations and muscle jerks. It is contraindicated in 
moderate-to-severe hypertension, hyperthyroidism, pregnancy and breastfeeding.

Methylphenidate and pemoline: These drugs have been the first-line drugs in the treatment of children (6). Pemoline, an oxazolidine derivative, can result in liver damage and, hence, is not readily available.

Modafinil: Modafinil, originally licensed for the treatment of patients with narcolepsy, is now also licensed for the treatment of EDS in patients with obstructive sleep apnea who continue to have symptoms despite adequate continuous positive airway pressure. It does not have any effect on cataplexy and other REM symptoms. The most important benefit of modafinil is that it has few side effects; there are no blood pressure side effects, it is not addictive and it has a low potential for abuse. The most common side effect is headache, and this can be reduced by a slow and progressive increase in the dosage. While amphetamines help to control cataplexy and sleep paralysis, modafinil has no effect on these symptoms; hence, on switching treatment from amphetamines to modafinil, there may be a recurrence of these symptoms. In patients already taking an anticataplectic drug simultaneously with modafinil, an increase in the daily dosage of this the anticataplectic medication may be needed.

\section{Drugs used for cataplexy}

Tricyclic antidepressants: Clomipramine, imipramine, protriptyline and desipramine have been used for cataplexy.

Monoamine oxidase inhibitors: Selegiline has been used for cataplexy, but it can result in dangerously high blood pressure. Selective serotonin reuptake inhibitors: Fluoxetine, sertraline and paroxetine have been used.

Serotonin and noradrenaline reuptake inhibitors: Venlafaxine lacks the sedative and antimuscarinic effects of the tricyclic antidepressants.

Sodium oxybate (gamma-hydroxybutyrate): Gammahydroxybutyrate is a naturally occurring metabolite of gammaaminobutyric acid found in the human nervous system (the highest concentrations are found in the hypothalamus and basal ganglia). It was discovered and first synthesized by Dr Henri-Marie Laborit, and is completely and rapidly metabolized into carbon dioxide and water. It is very fastacting, with maximum concentrations reaching in $35 \mathrm{~min}$ to $40 \mathrm{~min}$, and it has a terminal half-life of approximately $50 \mathrm{~min}$ (7). The possible therapeutic properties of sodium oxybate in humans were first suggested in 1960 (8). Since then, numerous studies have demonstrated that sodium oxybate produces clinical improvement in cataplexy and other symptoms related to narcolepsy. A four-week randomized, double-blind, placebocontrolled multicentre trial (9) showed that sodium oxybate in doses of $3 \mathrm{~g}, 6 \mathrm{~g}$ or $9 \mathrm{~g}$ taken in divided doses at bedtime and then at $2.5 \mathrm{~h}$ to $4 \mathrm{~h}$ later produced significant clinical improvements in narcolepsy symptoms. At doses of $6 \mathrm{~g}$ and $9 \mathrm{~g}$, there was a reduction in weekly cataplexy attacks (9). Following this study, a 12-month open-label, multicentre trial (10) of sodium oxybate in doses of $3 \mathrm{~g}$ to $9 \mathrm{~g}$ nightly produced improvements in narcolepsy symptoms, which were significant at four weeks and maximal after eight weeks. Headache, nausea, dizziness, somnolence and enuresis were reported as adverse effects (10).
Although little is known about its mechanism of action in narcolepsy, sodium oxybate produces subjective improvements in nocturnal sleep and daytime alertness. A recent study by Mamelak et al (11) concluded that nightly administration of sodium oxybate resulted in significant decreases in both subjective and objective measurements of daytime drowsiness. It also resulted in an initial increase in REM sleep, which was followed by a reduction of REM sleep as the dose of sodium oxybate increased. Lastly, they concluded that although the total nocturnal sleep time did not increase, there was an increase in slow-wave sleep, which could account for the improvement in EDS.

Apart from its use in narcolepsy, sodium oxybate may help patients with fibromyalgia (FM). In a double-blind, randomized, placebo-controlled crossover trial, Scharf et al (12) evaluated the effect of sodium oxybate on the symptoms of pain, fatigue and sleep quality in patients with FM. They concluded that sodium oxybate reduced the symptoms of pain and fatigue in patients with FM. FM is associated with alpha intrusion during sleep, which is accompanied by daytime complaints of musculoskeletal pain, fatigue and altered mood. Sodium oxybate dramatically reduced alpha intrusion in patients with FM.

Although the use of sodium oxybate may increase in the near future, the possibility of its misuse may also increase because it has gained wide recognition in the press as a 'recreational drug' used for date rape. It is tasteless and undetectable when mixed in a drink, and it creates a sense of amnesia when taken with alcohol; hence, it has been used as a date rape drug. Moreover, because the drug impairs their memory, victims may not seek help until days after the assault, at which time it is no longer detectable in the body.

\section{REFERENCES}

1. Gélineau J. De la narcolepsie. Gaz Hop (Paris) 1880;54:635-737.

2. Daniels L. Narcolepsy. Medicine 1934;13:1-122.

3. Guilleminault C, Mignot E, Grumet FC. Familial patterns of narcolepsy. Lancet 1989;2:1376-9.

4. Diagnostic Classification Steering Committee. The International Classification of Sleep Disorders: Diagnostic and Coding Manual. Minnesota: American Sleep Disorders Association, 1990.

5. Prinzmetal M, Bloomberg W. The use of benzedrine for the treatment of narcolepsy. JAMA 1935;105:2051-4.

6. Honda Y, Hishikawa Y. Effectiveness of pemoline in narcolepsy. Sleep Res 1970;8:192.

7. Scharf MB, Lai AA, Branigan B, Stover R, Berkowitz DB. Pharmacokinetics of gammahydroxybutyrate $(\mathrm{GHB})$ in narcoleptic patients. Sleep 1998;21:507-14.

8. Laborit H, Jouany JM, Gerard J, Fabiani F. Experimental study and clinical application of sodium hydroxybutyrate. Revue Agressologie 1960;1:397-406.

9. US Xyrem Multicenter Study Group. A randomized, double blind, placebo-controlled multicenter trial comparing the effects of three doses of orally administered sodium oxybate with placebo for the treatment of narcolepsy. Sleep 2002;25:42-9.

10. US Xyrem Multicenter Study Group. A 12-month, open label, multicenter extension trial with orally-administered sodium oxybate for the treatment of narcolepsy. Sleep 2003;26:31-5.

11. Mamelak M, Black J, Montplaisir J, Ristanovic R. A pilot study on the effects of sodium oxybate on sleep architecture and daytime alertness in narcolepsy. Sleep 2004;27:1327-34

12. Scharf MB, Baumann M, Berkowitz DV. The effects of sodium oxybate on clinical symptoms and sleep patterns in patients with fibromyalgia. J Rheumatol 2003;30:1070-4. 


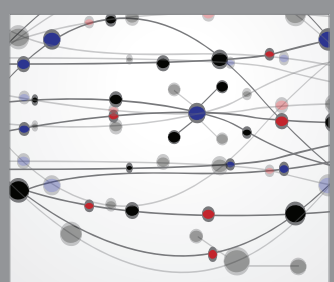

The Scientific World Journal
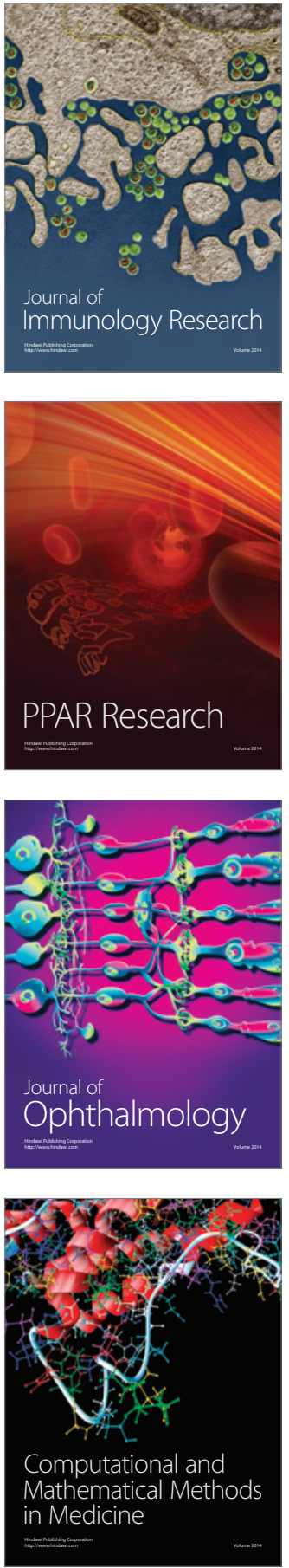

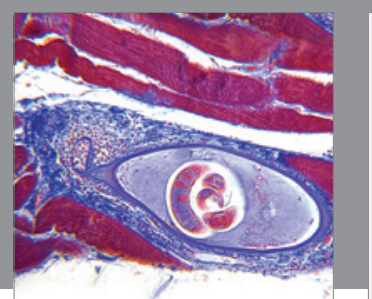

Gastroenterology Research and Practice

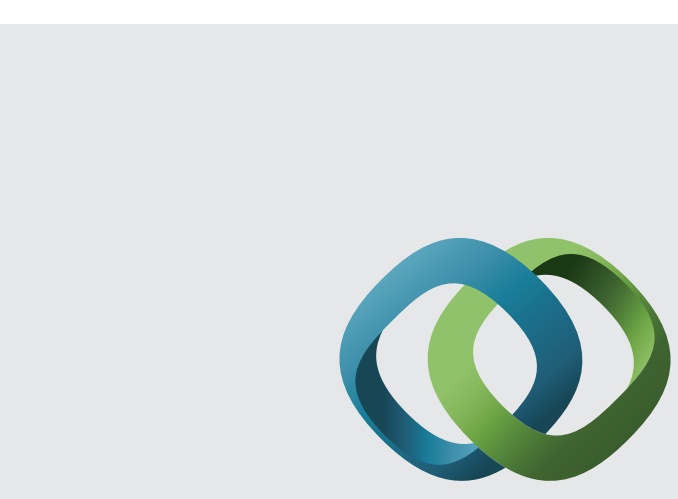

\section{Hindawi}

Submit your manuscripts at

http://www.hindawi.com
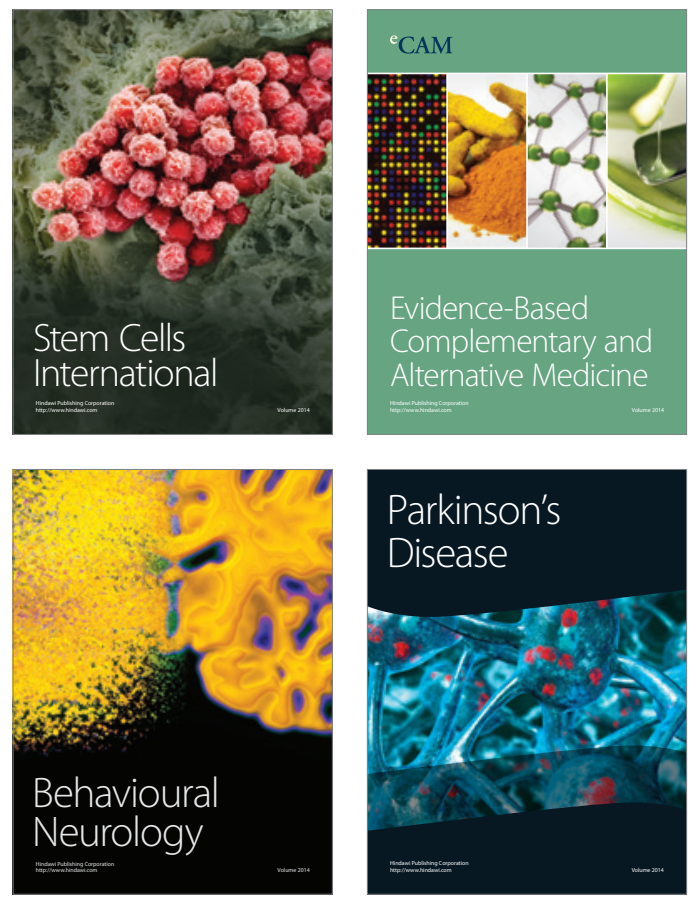
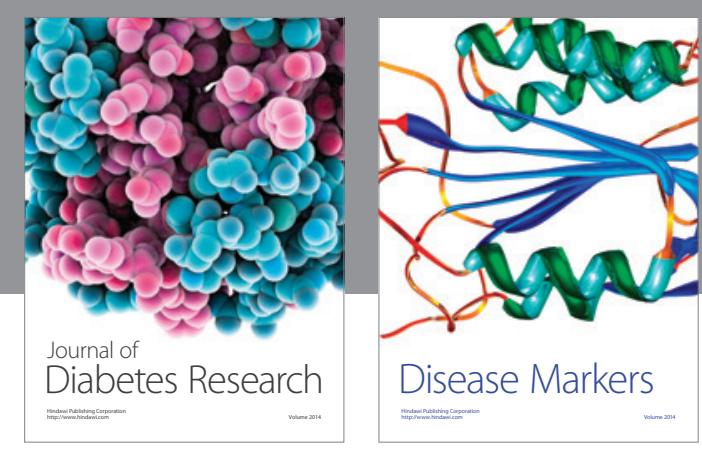

Disease Markers
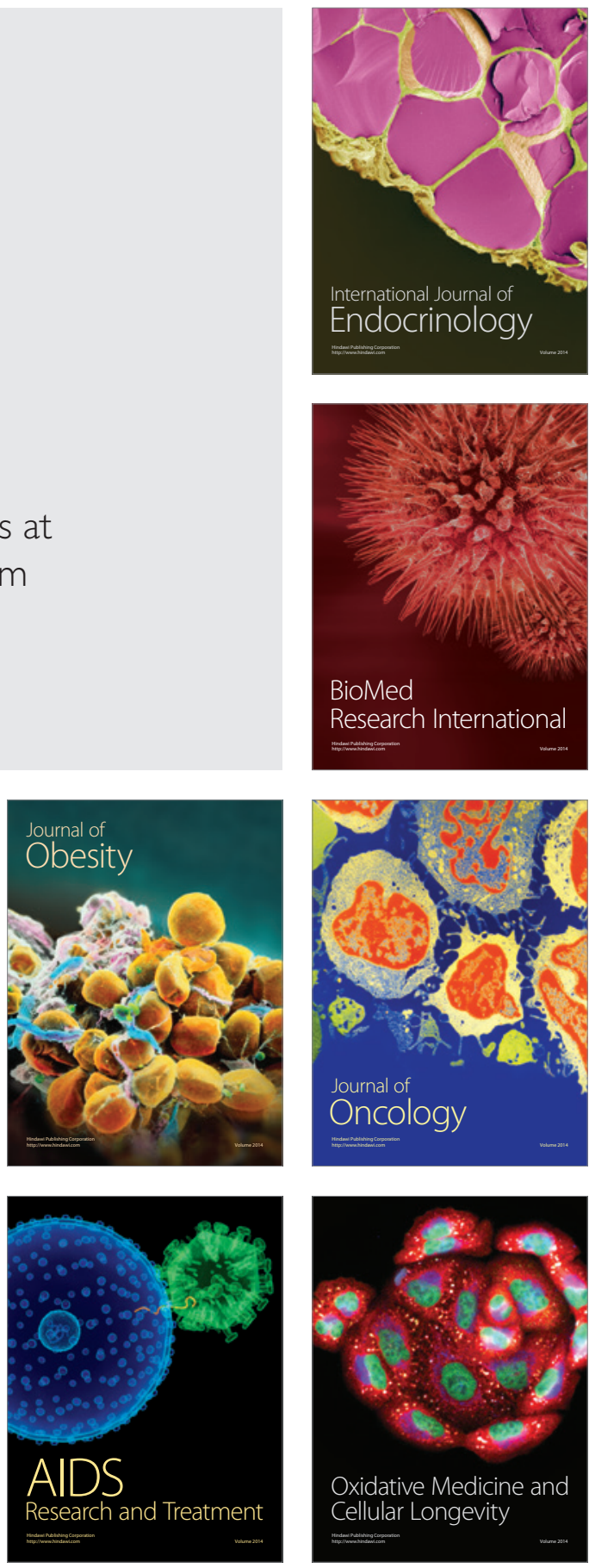